\title{
Arthrography of the temporomandibular joint: main diagnostic and therapeutic applications
}

\author{
Eva Levring Jäghagen ${ }^{1}\left[\right.$. Jan Ahlqvist ${ }^{1}(\mathbb{C}$
}

Received: 4 July 2019 / Accepted: 19 November 2019 / Published online: 20 December 2019

(c) The Author(s) 2019

\begin{abstract}
Arthrography is an alternative technique for diagnosing soft tissue derangements of the temporomandibular joint (TMJ) when magnetic resonance imaging (MRI) is not an option. This can be due to contraindications to perform a MRI on a specific patient or when MRI is not applicable for specific diagnoses of soft tissue derangements of the TMJ. It is an invasive method and can be used not only for diagnostics but also for therapeutic injections e.g. administration of corticosteroids
\end{abstract}

Keywords Fluoroscopy methods $\cdot$ Radiography $\cdot$ Temporomandibular joint disorders $\cdot$ Contrast media $\cdot$ Corticosteroids

\section{Quick reference/description}

Arthrography is an invasive technique for diagnosing temporomandibular joint (TMJ) soft tissue derangements. The most commonly used method for diagnosing TMJ soft tissue derangements is magnetic resonance imaging (MRI). However, MRI can not reveal conditions like adherences and perforations in the disc and posterior attachment, since the examination consists of still images. The functional examinations possible in arthrography are superior to the MRI "movies" for the same reason. Further, arthrography is the method of choice for soft tissue diagnostics when MRI is contra-indicated due to loose ferromagnetic objects in the body, metal objects like orthodontic devices in the head and neck region causing disturbances in the images or if the patient suffers from claustrophobia. Arthrography is also useful for guided therapeutic injections, e.g., administration of corticosteroids.

Eva Levring Jäghagen

eva.levring.jaghagen@umu.se

1 Oral and Maxillofacial Radiology, Department of Odontology, Umeå University, 90187 Umeå, Sweden 


\section{Indications}

In general practice, the findings before considering arthrography with or without administration of corticosteroid are:

- Clinical signs of TMJ arthralgia/arthritis, including pain on jaw movements and restricted jaw opening.

- No or minor pain relief after justified conservative treatment with either nonsteroid anti-inflammatory drugs, occlusal splint or, exercises of the jaw.

- Clinical signs of possible internal derangements and/or adherences.

Arthrography is useful for evaluation of the soft tissues of the TMJ to determine the position, configuration and dynamic function of the disc; and further, for verification of suspected adherences and/or perforations in the disc or posterior attachment and loose bodies in the TMJ. During an arthrography justified for other reasons, dynamic assessment of the articular disc position during use of an occlusal splint or intraoral orthotic device can be performed. Finally, guided intra-articular therapeutic injections with, e.g., corticosteroids can be included in the arthrography procedure.

\section{Contra-indications}

Previous reaction to iodine contrast media (can be overcome with corticosteroid premedication); anticoagulant medication or bleeding disorders; patient anxiety; and infection in the pre-auricular area.

\section{Materials/instruments}

To prepare before intervention according to Levring Jäghagen and Ahlqvist (2019):

- Chlorhexidine-ethyl alcohol

- Basin for disinfecting solution

- Gauze sponge

- Syringes

- Sodium hyaluronate

- Tube with needle

- Sterile arm sleeve

- Sterile gloves

- Sterile adhesive operation towel

- Surgical tape

- Sterile transparent film dressing

- Sterile adhesive drape and drape sheet

- Sterile plastic cap for the image intensifier

- Contrast medium

- Methylprednisolone acetate (Depo-Medrol) 
- Venous catheters

- Unrefined cotton wool

- Analgesics

\section{Procedure}

Fluoroscopy provides continuous X-ray imaging displayed on a monitor. The dose of radiation depends on the exposure parameters, mainly the exposure time, and the field of view. It allows detailed control during instrumentation and injection of various agents including contrast media.

\section{Contrast media for arthrography}

The administered contrast medium should have a high concentration of iodine (300 mg iodine/mL), since only small amounts of solution can be injected into the TMJ (1.5-2 mL). The risk of idiosyncratic reactions to the contrast medium is low as it is injected only into the joint compartments.

\section{Preparation of the patient before and after the examination}

Arthrography must be performed under highly antiseptic conditions. For detailed information about patient preparation and measures to take before and after the examination to prevent infection and other complications, please see Levring Jäghagen and Ahlqvist (2019).

\section{Positioning of the patient}

The examination is performed with the patient in a supine position, lying on the side with the TMJ of interest facing upwards. The patient's head is tilted slightly towards the table and rotated upwards. A vertical positioning of the $\mathrm{C}$-arm with the X-ray source under the exam table results in a lateral oblique transcranial projection of the upper TMJ (Fig. 1a, b). This projection prevents superimposition of the contralateral dense petrous part of the temporal bone on the opposing TMJ.

By tilting the X-ray source caudally, the central and medial parts of the TMJ are depicted (Fig. 2). Dynamic evaluation is performed in both projections. Differences in disc position between the lateral and medial parts of the TMJ imply a rotational component of a disc displacement.

\section{Single-contrast arthrography}

For conventional single-contrast arthrography, only one contrast medium is used, i.e., high attenuating iodine contrast media. Double-contrast technique requires a combination of high- and low-attenuating contrast media, it is complicated to 

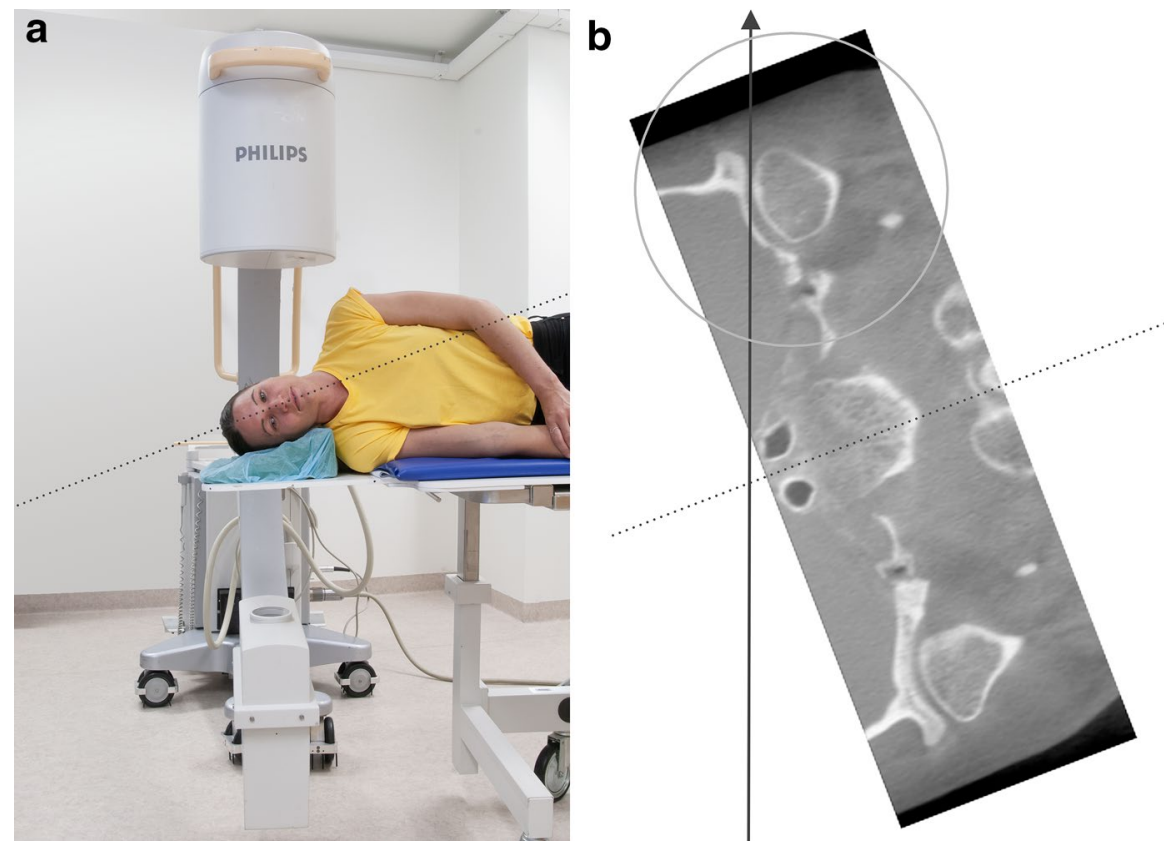

Fig. 1 a C-arm alignment for an oblique transcranial projection illustrated as in a clinical set-up. b The coronal slice from a CBCT examination of the TMJ illustrates how the lateral parts of the joint and components will be imaged with this projection

perform, require longer exposure time and operator experience; therefore, the technique is rarely utilized. Single-contrast technique is performed as follows:

\section{Anesthesia}

Anesthesia is administered prior to administration of contrast media into the TMJ. Under fluoroscopy, the needle tip is positioned on the skin in an appropriate position to ensure accurate insertion (Fig. 3a). It is inserted in line with the central beam towards the posterior aspect of the lateral pole of the condyle. The anesthesia is injected while slowly retracting the needle to the skin surface. Anesthesia is also deposited in the region anteriorly to the condyle targeting the posterior aspect of the articular tubercle for access to the upper joint compartment (Fig. 3b).

\section{Injection of contrast media}

Prior to administration of contrast media, elimination of air bubbles from the syringe and the tube with needle is essential to avoid confusion when diagnosing. The positioning of the needle for injection into the TMJ compartments is similar to that of anesthesia. A slight increase in resistance followed by decrease during advancement of the needle implies penetration through the joint capsule into the joint compartment. A correct intra-articular position of the needle is confirmed by fluoroscopy 


\section{a}

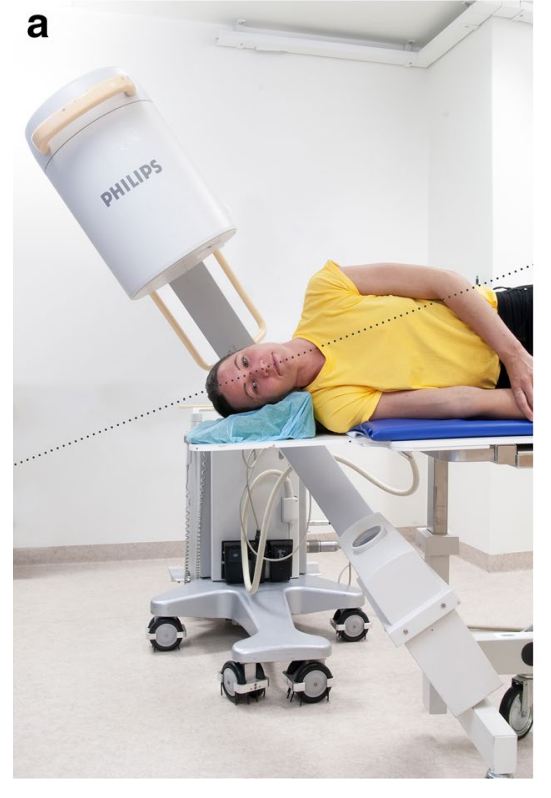

b

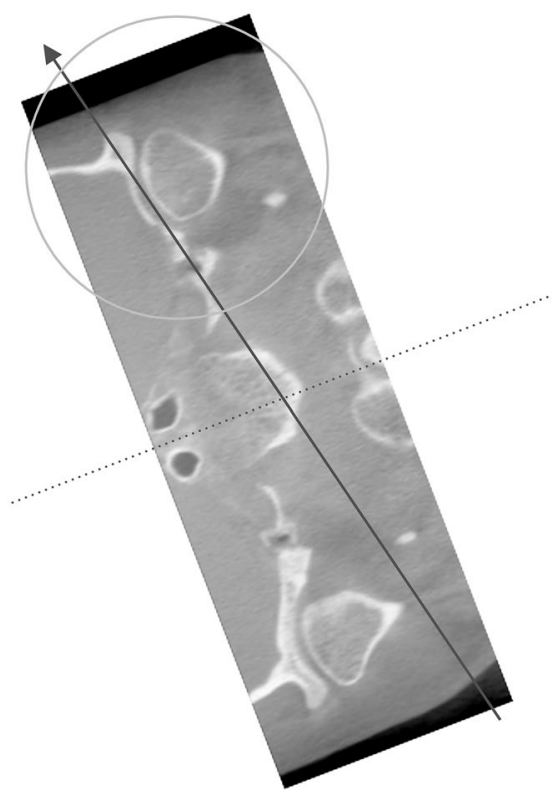

Fig. 2 a C-arm alignment for depiction of the medial part of the left TMJ shown in the clinical set-up. b The projection illustrated in a coronal slice from a CBCT examination

when the injected contrast medium is rapidly distributed into the joint compartment. If the needle has an incorrect position, e.g., in the soft tissue surrounding the TMJ, the injection leads to a diffuse appearance in the fluoroscopic image, and the needle position needs to be adjusted. To minimize damage to the condyle and tubercle, the bevel of the needle should be faced towards the joint surfaces during cannulation.
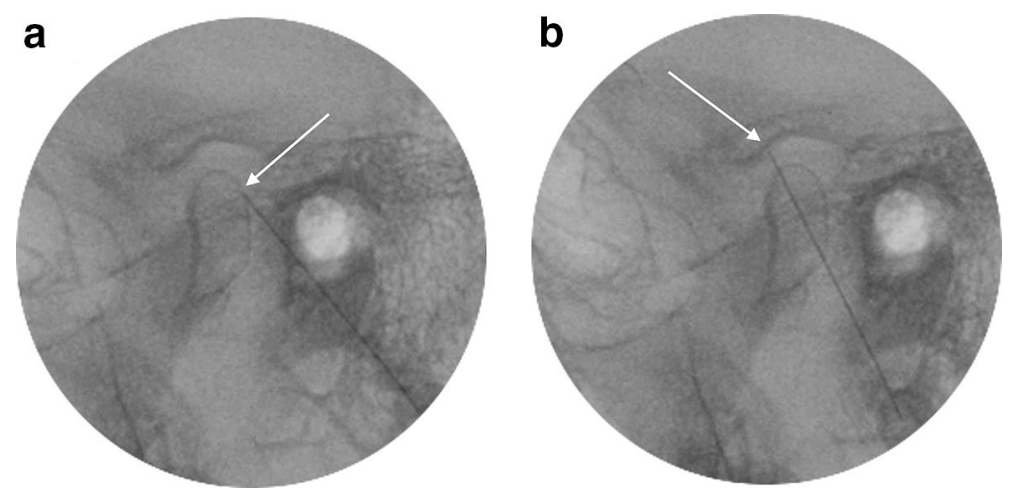

Fig. 3 Needle positioning during injection of anesthesia prior to arthrography of the TMJ (left side). a Needle tip positioned in close relation to the upper posterior part of the lateral pole of the condyle for administration of anesthesia. b Needle positioned in close relation to the posterior surface of the articular tubercle for administration of anesthesia prior to contrast injection into the upper joint compartment 

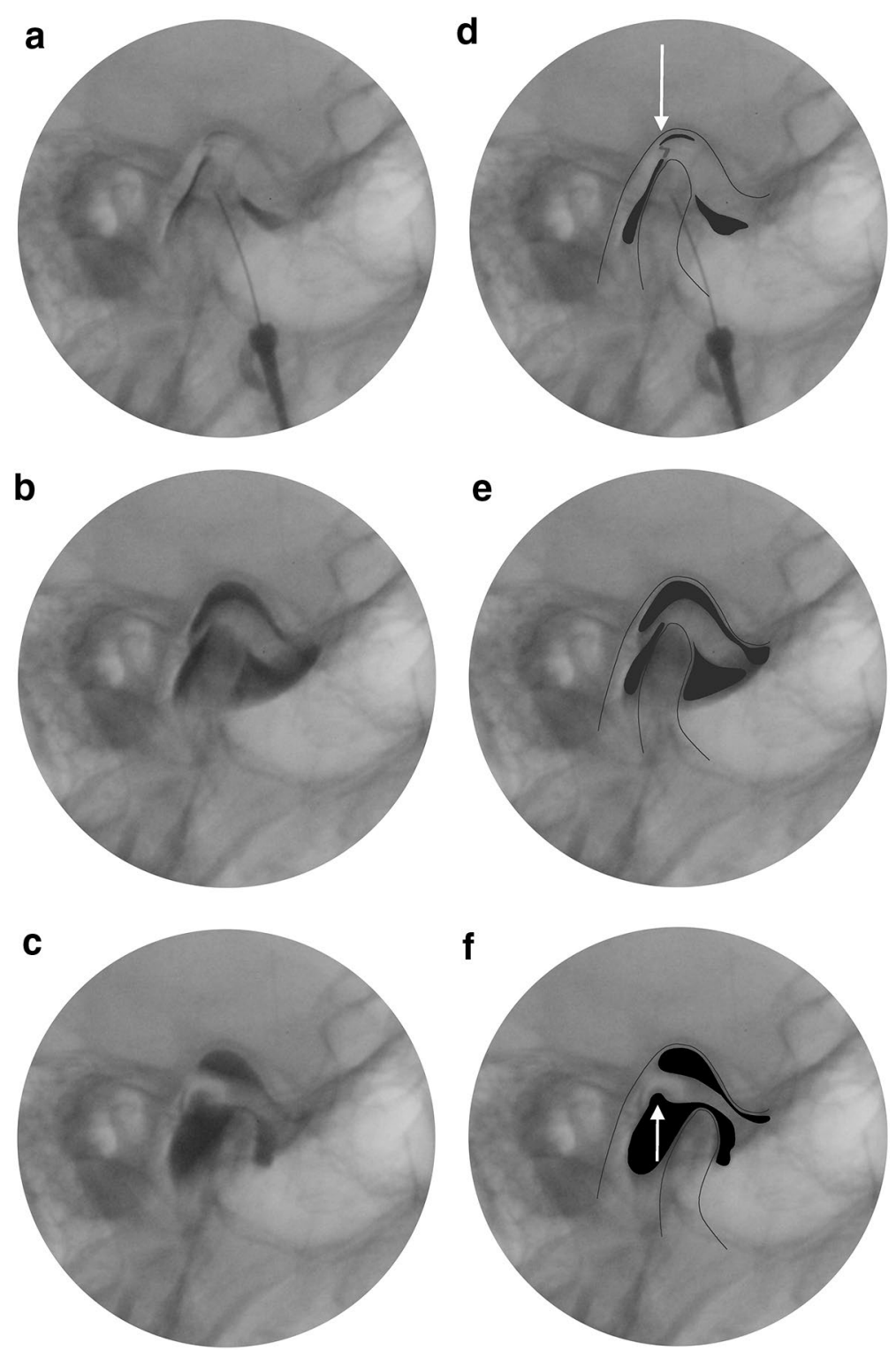

Fig. 4 Arthrography of the TMJ (right side) showing anterior disc displacement with reduction and perforation of the posterior attachment. a-c Oblique transcranial projection revealing the lateral part of the joint. d-f Corresponding images where the contrast media are highlighted and the joint components outlined. a, d Contrast media leaking into the upper compartment via a perforation in the posterior attachment of the disc in closed mouth position (arrow). b, e Anterior disc displacement, both compartments loaded with contrast in closed mouth position. c, f The disc is reduced and a defect in the posterior attachment is visualized in open mouth position (arrow) 


\section{Diagnostics using arthrography}

\section{Disc displacement with and without reduction}

The disc is visualized between the joint compartments after they are filled with contrast media. The shape and position of the disc are first assessed in a closed mouth position. Deformity of the disc can range from slight to extensive. The sagittal projection allows assessment of anterior disc displacement in relation to the condyle. During opening of the mouth, the disc either reduces or is pushed forward in a permanently displaced position showing disc displacement without reduction.

\section{Adhesions}

An immobile disc in relation to the condyle, the temporomandibular fossa or the tuberculum indicates adhesion.
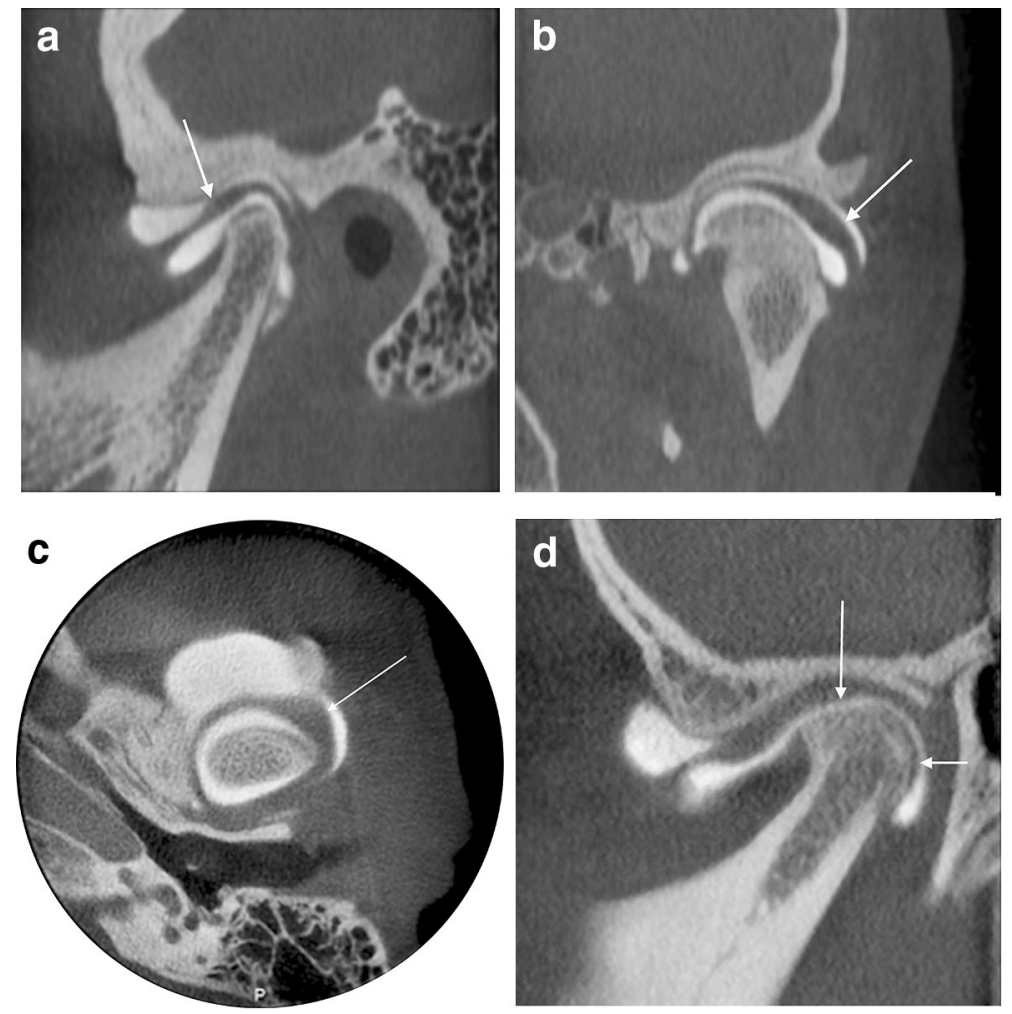

Fig. 5 Arthrotomography of the TMJ (left side). a The disc is anteriorly displaced with the posterior band (arrow) placed anterior to the condyle, $\mathbf{b}$ the disc is laterally displaced (arrow) in the coronal plane and in $\mathbf{c}$ the axial plane the anterolateral disc displacement (arrow) is depicted, $\mathbf{d}$ sagittal plane depicting medial part of the joint, showing contrast media in direct contact with the bone superior on the condyle indicating loss of cartilage (long arrow) contrary to the posterior part of the caput showing the cartilage as a gap between the bone and the contrast media (short arrow) 


\section{Perforation/rupture of the disc or posterior attachment}

Leakage of contrast media into the upper compartment during administration of contrast media into the lower joint compartment indicates perforation in the disc or its posterior attachment (Fig. 4).

\section{Evaluation of occlusal splint function}

The function of an occlusal splint designed to keep the disc in a correct position in patients with disc displacement with reduction can be verified during the examination with arthrography. The splint is positioned in the mouth after maximum mouth opening to ensure that disc reduction has been obtained. The disc position and function during jaw movement with the occlusal splint in position should then be normal.

\section{Arthrotomography}

Arthrography immediately followed by CBCT imaging, i.e., arthrotomography of the TMJ enables detailed visualization of the disc position in three planes (Fig. 5). Arthrotomography also optimizes detection and visualization of adhesions, small loose bodies in the joint, and loss of cartilage on the condyle (Fig. 5d) and/or temporal bone.

\section{Arthrography-guided therapeutic injections with corticosteroids}

Corticosteroids are used for treatment of TMJ pain. They are useful for patients with TMJ arthritis-related symptoms or MRI-verified signs of TMJ inflammation. Arthrography facilitates guided intra-articular corticosteroid injections when combined with contrast media. The proportion of contrast media in relation to the corticosteroid can vary. The recommended maximum dosage of corticosteroids injected into the TMJ should not be exceeded. For methylprednisolone acetate the maximum dosage for TMJ is $40 \mathrm{mg}$.

Injection of anesthetics into the TMJ before the arthrography can be used as a diagnostic tool when differentiation between TMJ pain and, e.g., muscular or ear pain is requested. If pain relief is obtained following the injection, the TMJ is most likely the source of pain.

\section{Pitfalls and complications}

- Arthrography is an invasive method and requires the use of ionizing radiation. Making a reliable diagnosis regarding sideway disc displacement without CBCT examination is difficult.

- Fluid injection into the joint can impair joint function during the examination. Multiple attempts to locate the compartments can cause iatrogenic perforation of the disc or posterior attachment. 
- Local complications of intra-articular injections include bleeding and pain.

- In case of corticosteroid injections, transient increase in pain (steroid flare), mild headache and facial flushing can occur. Patients with diabetes can experience a short spike in blood glucose level.

- While administering local anesthesia, a risk of anesthetizing other unintended nerves is present, particularly the facial nerve resulting in transient facial nerve palsy with an inability of eyelid closure on the affected side. This risk is proportional to the injected amount of anesthesia. Joint inflammation can cause local anesthetic failure.

Acknowledgements Open access funding provided by Umea University. We would like to express our sincere gratitude to Dr. Per Erik Legrell and research engineer Magnus Johansson for their contributions to the illustrations.

Open Access This article is licensed under a Creative Commons Attribution 4.0 International License, which permits use, sharing, adaptation, distribution and reproduction in any medium or format, as long as you give appropriate credit to the original author(s) and the source, provide a link to the Creative Commons licence, and indicate if changes were made. The images or other third party material in this article are included in the article's Creative Commons licence, unless indicated otherwise in a credit line to the material. If material is not included in the article's Creative Commons licence and your intended use is not permitted by statutory regulation or exceeds the permitted use, you will need to obtain permission directly from the copyright holder. To view a copy of this licence, visit http://creativecommons.org/licen ses/by/4.0/.

\section{Further reading}

1. Levring Jäghagen E, Ahlqvist J (2019) Chapter 16, arthrography of the temporomandibular joint and arthrography-guided steroid treatment. In: Rozylo-Kalinowska I, Orhan K (eds) Imaging of the temporomandibular joint. Springer Nature Switzerland AG. https://doi.org/10.1007/978-3-319-99468-0_16. (ISBN 978-3-319-99467-3)

2. Ahlqvist J, Isberg A (2001) Chapter 16, Radiographic imaging. In: Isberg A (ed) Temporomandibular joint dysfunction: a practitioner's guide. ISIS Medical Media Ltd, Oxford, p 187. (ISBN 1901865444)

3. Stoustrup P, Kristensen KD, Verna C, Kuseler A, Pedersen TK, Herlin T (2013) Intra-articular steroid injections for temporomandibular joint arthritis in juvenile idiopathic arthritis: a systematic review on efficacy and safety. Semin Arthritis Rheum 43(1):63-70

4. Stojanovic MP, Vu T-N, Caneris O, Slezak J, Cohen SP, Sang CN (2002) The role of fluoroscopy in cervical epidural steroid injections. Spine 27(5):509-514

5. Habib GS (2009) Systemic effects of intra-articular corticosteroids. Clin Rheumatol 28(7):749-756

6. Ahlqvist J, Legrell PE (1993) A technique for the accurate administration of corticosteroids in the temporomandibular joint. Technical report. DMFR 22:211-213

Publisher's Note Springer Nature remains neutral with regard to jurisdictional claims in published maps and institutional affiliations. 